\title{
THE GYRO-SYNCHROTRON RADIATION FROM MOVING TYPE IV SOURCES IN THE SOLAR CORONA
}

\author{
G. A. DULK \\ Division of Radiophysics, CSIRO, Sydney, Australia
}

\begin{abstract}
Solar Phys.). Calculations of the gyro-synchrotron emission are made for conditions which might be expected in moving type IV sources in the solar corona. Two simple models for an evolving source are treated: a uniform cube and an inhomogeneous sphere. The results suggest that most moving sources have the following features: (1) A rather strong magnetic field, $\approx 10 \mathrm{G}$, is carried out within the source. This is required to achieve the high degree of circular polarization often observed. (2) Synchrotron self-absorption causes the source to be optically thick at frequencies less than about $100 \mathrm{MHz}$, thus restricting the bandwidth of the radiation. The self-absorption decreases as the source moves outward and expands. The turnover frequency, which separates the optically thick and thin spectral regimes, moves rapidly to lower frequencies, accompanied by a change from low to high circular polarization. In the case of an inhomogeneous source, the source appears to be larger at the lower frequencies. (3) Razin-Tsytovich suppression cannot be an important factor in determining the characteristics of most sources.
\end{abstract}

\section{DISCUSSION}

Rosenberg: A plasma blob with such a strong magnetic field would have to be self-contained by the field; hence, the field should be quite tangled up. How would that effect the radiation and in particular the polarization?

Dulk: The degree of polarization would go down drastically. Thus, we require a well-ordered nearly homogeneous field with few meanderings or inclusions with the opposite polarity.

Smith: In the 'westward ho' event we have evidence of a very coherent field since both polarizations are seen. Have you applied your calculations to each source in this event?

Dulk: If we take a model of a toroidal magnetic field of about $10 \mathrm{G}$, the double source with opposite polarizations could be explained. By inverting a toroid, i.e. introducing a ring current with its poloidal field, we could end up with a single source in the center of the ring where the field is high and the particles radiate efficiently.

Kane: What are the ion and electron densities inside the blob?

Dulk: In the two models I have considered we have

\begin{tabular}{lcll} 
& Model 1 & Model 2 & \\
\hline$B$ & 3.4 & 13.6 & $\mathrm{G}$ \\
$N_{T}$ & $10^{35}$ & $10^{33}$ & electrons with energy $>10 \mathrm{keV}$ \\
$n$ & $10^{5}$ & $10^{3}$ & $\mathrm{~cm}^{-3}$ \\
$m_{c}$ & $\$ 0.5$ & $\$ 0.8$ & degree of circular polarization \\
\hline
\end{tabular}

* Exchange visitor from the Dept. of Astro-Geophysics, University of Colorado, Boulder, Colo., U.S.A. 
Mangeney: If you consider the case of a ring current, you should also have two sources; since particles will remain in the external regions where the magnetic field is lower/longer than inside.

Dulk: It depends on the distribution of the particles. If most of them have pitch angles to allow them to thread through the center, they will radiate strongly only in the strong field near the center. On the other hand, if you put all the particles in the outer regions, with small pitch angles such as are in the Earth's radiation belts, the source will be a ring with radiation of the opposite polarization. In between these cases you might have a central source of one polarization surrounded by a ring of opposite polarization. The latter kind has not been observed.

Schmidt: I take it that you are well aware about the problem caused by a field strength of $13 \mathrm{G}$ in this environment. I think the timescales are too long to balance the surplus pressure by inertia. Would you like to comment on this problem?

Dulk: It is definitely a problem but the large field seems to be required to give the large degree of polarization observed. The configuration is not stable but relaxes with a typical time of a few minutes to a few tens of minutes. Even if there were no plasma inside the source, the field could not be contained by the surrounding corona. So we must assume that forces internal to the plasmoid, such as magnetic tension in field loops, will prevent it from deteriorating too rapidly. I refer to the paper by Altschuler, Lilliequist, and Nakagawa, who investigated the stability of a toroid under photospheric conditions. It is an open question what happens to such a source in the corona.

Brown: I would just like to add that if you were to associate these sources with Frost's second phase hard X-rays, the $10^{27}$ erg you require would have to be increased by several orders (in the $10-100 \mathrm{keV}$ particle range). This would make the energy and pressure problems much worse.

Dulk: I wouldn't suggest such as association. 